\title{
Eigenvalue Bounds for a Class of Schrödinger Operators in a Strip
}

\author{
Martin Karuhanga $(\mathbb{D}$ \\ Department of Mathematics, Mbarara University of Science and Technology, P.O. BOX 1410, Mbarara, Uganda \\ Correspondence should be addressed to Martin Karuhanga; mkaruhanga@must.ac.ug
}

Received 9 July 2018; Accepted 22 November 2018; Published 12 December 2018

Academic Editor: Sivaguru Sritharan

Copyright (c) 2018 Martin Karuhanga. This is an open access article distributed under the Creative Commons Attribution License, which permits unrestricted use, distribution, and reproduction in any medium, provided the original work is properly cited.

\begin{abstract}
This paper is concerned with the estimation of the number of negative eigenvalues (bound states) of Schrödinger operators in a strip subject to Neumann boundary conditions. The estimates involve weighted $L^{1}$ norms and $L \ln L$ norms of the potential. Estimates involving the norms of the potential supported by a curve embedded in a strip are also presented.
\end{abstract}

\section{Introduction}

The celebrated Cwikel-Lieb-Rozenblum (CLR) inequality [1] gives an upper bound for the number of negative eigenvalues of the Schrödinger operator $-\Delta-V, V \geq 0$ on $L^{2}\left(\mathbb{R}^{d}\right), d>2$. It is known that the CLR inequality does not hold for $d=2$ and one of the reasons is that the Sobolev space $H^{1}\left(\mathbb{R}^{2}\right)$ is not continuously embedded in $L^{\infty}\left(\mathbb{R}^{2}\right)$. However, $H^{1}\left(\mathbb{R}^{2}\right) \hookrightarrow$ $L^{q}\left(\mathbb{R}^{2}\right)$ for all $q \in[2,+\infty)$ and there are estimates involving $\int_{\mathbb{R}^{2}}|V(x)|^{r} d x, \forall r>1$ (see, e.g., [2-4]). More precisely, $H^{1}\left(\mathbb{R}^{2}\right)$ is embedded in a space of exponentially integrable functions which lies between $L^{1}\left(\mathbb{R}^{2}\right)$ and $L^{p}\left(\mathbb{R}^{2}\right), p>1$ (see, e.g., [5]). This gives rise to estimates involving a norm of $V$ weaker than $\|V\|_{L^{r}}, r>1$, namely, the Orlicz $L \ln L$ norm (see, e.g., $[6,7]$ ). The strongest known estimates have been obtained in [6]. For more information regarding upper estimates for the number of negative eigenvalues of two-dimensional Schrödinger operators refer to [2-4, 6-9] and the references therein. This paper provides estimates for the number of negative eigenvalues of the Schrödinger operator $-\Delta-V$ on $L^{2}(S)$ whose domain is characterized by the Neumann boundary conditions, where $S$ is an infinite straight strip. We use the results of Shargorodsky [6] to obtain improved versions of the estimates by Grigor'yan and Nadirashvili [3]. This improvement is achieved by replacing $\|V\|_{L^{p}}, p>1$ in the estimates of $[3$, Section 7] by the $L \ln L$ norms of $V$. In addition, these estimates are extended to the case of strongly singular potentials (see Section 4).
The precise description of the operator here studied is as follows.

Let $S:=\left\{\left(x_{1}, x_{2}\right) \in \mathbb{R}^{2}: x_{1} \in \mathbb{R}, 0<x_{2}<a\right\}, a>0$ and $V: \mathbb{R}^{2} \longrightarrow \mathbb{R}$ be a function integrable on bounded subsets of $S$. Consider the following self-adjoint operator on $L^{2}(S)$ :

$$
H_{V}=-\Delta-V, \quad V \geq 0,
$$

with homogeneous Neumann boundary conditions both at $x_{2}=0$ and $x_{2}=a$. The main objective of this paper is to obtain estimates for the number of negative eigenvalues of (1) in terms of the norms of $V$.

The strategy used here is as follows: The problem is split into two problems. The first one is defined by the restriction of the quadratic form associated with operator (1) to the subspace of functions of the form $w\left(x_{1}\right) u_{1}\left(x_{2}\right)$, where $u_{1}$ is the first eigenfunction of the one-dimensional differential operator on $L^{2}((0, a))$ and, hence, is reduced to a well studied one-dimensional Schrödinger operator with the potential equal to a weighted mean value $\widetilde{V}$ of $V$ over $(0, a)$. The second problem is defined by a class of functions orthogonal to constant functions in the $L^{2}((0, a))$ inner product.

\section{Preliminaries}

Let $(\Omega, \Sigma, \mu)$ be a measure space and let $\Psi:[0,+\infty) \longrightarrow$ $[0,+\infty)$ be a nondecreasing function. The Orlicz class $K_{\Psi}(\Omega)$ is the set of all of measurable functions $f: \Omega \longrightarrow \mathbb{C}($ or $\mathbb{R})$ such that 


$$
\int_{\Omega} \Psi(|f(x)|) d \mu(x)<\infty
$$

If $\Psi(t)=t^{p}, 1 \leq p<\infty$, this is just the $L^{p}(\Omega)$ space. The Orlicz space $L_{\Psi}(\Omega)$ is the linear span of the Orlicz class $K_{\Psi}(\Omega)$, that is, the smallest vector space containing $K_{\Psi}(\Omega)$.

Definition 1. A continuous nondecreasing convex function $\Psi:[0,+\infty) \longrightarrow[0,+\infty)$ is called an $N$-function if

$$
\begin{aligned}
\lim _{t \rightarrow 0+} \frac{\Psi(t)}{t} & =0 \\
\text { and } \lim _{t \rightarrow \infty} \frac{\Psi(t)}{t} & =\infty .
\end{aligned}
$$

The function $\Phi:[0,+\infty) \longrightarrow[0,+\infty)$ defined by

$$
\Phi(t):=\sup _{s \geq 0}(s t-\Psi(s))
$$

is called complementary to $\Psi$.

Definition 2. An $N$-function $\Psi$ is said to satisfy a global $\Delta_{2}$ condition if there exists a positive constant $k$ such that, for every $t \geq 0$,

$$
\Psi(2 t) \leq k \Psi(t) .
$$

Similarly $\Psi$ is said to satisfy a $\Delta_{2}$-condition near infinity if there exists $t_{0}>0$ such that (5) holds for all $t \geq t_{0}$.

Let $\Phi$ and $\Psi$ be mutually complementary $N$-functions, and let $L_{\Phi}(\Omega)$ and $L_{\Psi}(\Omega)$ be the corresponding Orlicz spaces. We will use the following norms on $L_{\Psi}(\Omega)$ :

$$
\|f\|_{\Psi}=\|f\|_{\Psi, \Omega}=\sup \left\{\left|\int_{\Omega} f g d \mu\right|: \int_{\Omega} \Phi(|g|) d \mu \leq 1\right\}
$$

and

$$
\begin{aligned}
\|f\|_{(\Psi)} & =\|f\|_{(\Psi, \Omega)} \\
& =\inf \left\{\kappa>0: \int_{\Omega} \Psi\left(\frac{|f|}{\kappa}\right) d \mu \leq 1\right\} .
\end{aligned}
$$

These two norms are equivalent:

$$
\|f\|_{(\Psi)} \leq\|f\|_{\Psi} \leq 2\|f\|_{(\Psi)}, \quad \forall f \in L_{\Psi}(\Omega)
$$

(see [5]).

Note that

$$
\int_{\Omega} \Psi\left(\frac{|f|}{\kappa_{0}}\right) d \mu \leq C_{0}, \quad C_{0} \geq 1 \Longrightarrow\|f\|_{(\Psi)} \leq C_{0} \kappa_{0} .
$$

It follows from (9) with $\kappa_{0}=1$ that

$$
\|f\|_{(\Psi)} \leq \max \left\{1, \int_{\Omega} \Psi(|f|) d \mu\right\} .
$$

We will need the following equivalent norm on $L_{\Psi}(\Omega)$ with $\mu(\Omega)<\infty$ which was introduced in [7]:

$$
\begin{aligned}
\|f\|_{\Psi}^{(\text {av })} & =\|f\|_{\Psi, \Omega}^{(\text {av) }} \\
& =\sup \left\{\left|\int_{\Omega} f g d \mu\right|: \int_{\Omega} \Phi(|g|) d \mu \leq \mu(\Omega)\right\} .
\end{aligned}
$$

We will use the following pair of pairwise complementary $N$ functions:

$$
\begin{aligned}
& \mathscr{A}(s)=e^{|s|}-1-|s|, \\
& \mathscr{B}(s)=(1+|s|) \ln (1+|s|)-|s|,
\end{aligned}
$$

$s \in \mathbb{R}$.

Let $I_{1}, I_{2} \subseteq \mathbb{R}$ be nonempty open intervals. We denote by $L_{1}\left(I_{1}, L_{\mathscr{B}}\left(I_{2}\right)\right)$ the space of measurable functions $f: I_{1} \times$ $I_{2} \longrightarrow \mathbb{C}$ such that

$$
\|f\|_{L_{1}\left(I_{1}, L_{\mathscr{B}}\left(I_{2}\right)\right)}:=\int_{I_{1}}\left\|f\left(x_{1}, \cdot\right)\right\|_{\mathscr{B}, I_{2}} d x_{1}<+\infty .
$$

Let us recall that a sequence $\left\{a_{n}\right\}$ belongs to the "weak $l_{1}$ space" (Lorentz space) $l_{1, w}$ if the quasinorm

$$
\left\|\left\{a_{n}\right\}\right\|_{1, w}=\sup _{s>0}\left(s \operatorname{card}\left\{n:\left|a_{n}\right|>s\right\}\right)
$$

is finite. It is a quasinorm in the sense that it satisfies the weak version of the triangle inequality:

$$
\left\|\left\{a_{n}\right\}+\left\{b_{n}\right\}\right\|_{1, w} \leq 2\left(\left\|\left\{a_{n}\right\}\right\|_{1, w}+\left\|\left\{b_{n}\right\}\right\|_{1, w}\right) .
$$

Quasinorm (14) induces a topology on $l_{1, w}$ in which this space is nonseparable. The closure of the set of elements $a_{n}$ with only finite number of nonzero terms is a separable subspace in $l_{1, w}$. It is well known that $l_{1} \subset l_{1, w}$ and

$$
\left\|\left\{a_{n}\right\}\right\|_{1, w} \leq\left\|\left\{a_{n}\right\}\right\|_{l_{1}}
$$

(see, e.g., [10] for more details).

\section{Estimating the Number of Negative Eigenvalues in a Strip}

Define (1) via its quadratic form

$$
\begin{aligned}
q_{V, S}[u] & :=\int_{S}|\nabla u(x)|^{2} d x-\int_{S} V(x)|u(x)|^{2} d x, \\
\operatorname{dom}\left(q_{V, S}\right) & =W_{2}^{1}(S) \cap L^{2}(S, V d x),
\end{aligned}
$$

where $W_{2}^{1}(S)$ denotes the standard Sobolev space $H^{1}(S)$. Let $N_{-}\left(q_{V, S}\right)$ denote the number of negative eigenvalues of (1) repeated according to their multiplicities. Then $N_{-}\left(q_{V, S}\right)$ is given by

$$
N_{-}\left(q_{V, S}\right)=\sup _{L}\left\{\operatorname{dim} L: q_{V, S}[u]<0, \forall u \in L \backslash\{0\}\right\},
$$

where $L$ denotes a linear subspace of $\operatorname{dom}\left(q_{V, S}\right)$ (see, e.g., [10, Theorem 10.2.3]).

Let

$$
\begin{aligned}
& I_{n}:=\left[2^{n-1}, 2^{n}\right], \quad n>0, \\
& I_{0}:=[-1,1], \\
& I_{n}:=\left[-2^{|n|},-2^{|n|-1}\right], \quad n<0,
\end{aligned}
$$


and

$$
\begin{aligned}
& \mathscr{G}_{n}:=\int_{I_{n}} \int_{0}^{a}\left|x_{1}\right| V(x) d x, \quad n \neq 0, \\
& \mathscr{G}_{0}:=\int_{I_{0}} \int_{0}^{a} V(x) d x .
\end{aligned}
$$

Furthermore, let

$$
\begin{aligned}
J_{n} & :=(n, n+1), \quad n \in \mathbb{Z}, \\
I & :=(0, a) \\
\text { and } \mathscr{D}_{n} & :=\|V\|_{L_{1}\left(J_{n}, L_{\mathscr{B}}(I)\right)} .
\end{aligned}
$$

Then we have the following result.

Theorem 3. There exist constants $c, C>0$ such that

$$
\begin{array}{r}
N_{-}\left(q_{V, S}\right) \leq 1+C\left(\sum_{\left\{n \in \mathbb{Z}, \mathscr{G}_{n}>c\right\}} \sqrt{\mathscr{G}_{n}}+\sum_{\left\{n \in \mathbb{Z}, \mathscr{D}_{n}>c\right\}} \mathscr{D}_{n}\right), \\
\forall V \geq 0 .
\end{array}
$$

Let

$$
L_{1}:=\left\{u \in L^{2}(S): u(x)=u\left(x_{1}\right)\right\}
$$

and $P: L^{2}(S) \longrightarrow L_{1}$ be a projection defined by

$$
P v(x):=\frac{1}{a} \int_{0}^{a} v(x) d x_{2}=P v\left(x_{1}\right) .
$$

Indeed, $P$ is a projection since $P^{2}=P$. Let $L_{2}:=(I-P) L^{2}(S)$, and then one can show that $L^{2}(S)=L_{1} \oplus L_{2}$. Here and below $\oplus$ denotes a direct orthogonal sum.

Indeed for all $v \in L_{2}$ we have

$$
\begin{aligned}
\int_{0}^{a} v(x) d x_{2} & =\int_{0}^{a}(I-P) v(x) d x_{2} \\
& =\int_{0}^{a} v(x) d x_{2}-\int_{0}^{a} P v(x) d x_{2} \\
& =\int_{0}^{a} v(x) d x_{2}-\int_{0}^{a} P v\left(x_{1}\right) d x_{2} \\
& =\int_{0}^{a} v(x) d x_{2}-\int_{0}^{a} v(x) d x_{2}=0 .
\end{aligned}
$$

Now pick $w \in L_{1}$ and $v \in L_{2}$, then,

$$
\begin{aligned}
\langle v, w\rangle_{L^{2}(S)} & =\int_{S} v(x) \overline{w\left(x_{1}\right)} d x \\
& =\int_{\mathbb{R}}\left(\int_{0}^{a} v(x) d x_{2}\right) \overline{w\left(x_{1}\right)} d x_{1}=0 .
\end{aligned}
$$

Similarly, let

$$
\mathscr{H}_{1}:=\left\{u \in W_{2}^{1}(S): u(x)=u\left(x_{1}\right)\right\}
$$

and $\mathscr{H}_{2}:=(I-P) W_{2}^{1}(S)$, and then

$$
W_{2}^{1}(S)=\mathscr{H}_{1} \oplus \mathscr{H}_{2} .
$$

Indeed, for all $v \in \mathscr{H}_{1}$ and all $w \in \mathscr{H}_{2}$ we have

$$
\begin{aligned}
& \langle v, w\rangle_{W_{2}^{1}(S)}=\int_{S}\left(v\left(x_{1}\right) \overline{w(x)}+v_{x_{1}}\left(x_{1}\right) \overline{w_{x_{1}}(x)}\right. \\
& \left.\quad+v_{x_{2}}\left(x_{1}\right) \overline{w_{x_{2}}(x)}\right) d x=0 .
\end{aligned}
$$

This is so because $v, v_{x_{1}} \in L_{1}, \bar{w}, \overline{w_{x_{1}}} \in L_{2}$, and $v_{x_{2}}=0$. To see this note that $v\left(x_{1}\right)$ and $v_{x_{1}}\left(x_{1}\right)$ do not depend on $x_{2}$ implying that $v_{x_{1}} \in L_{1}$. Also, $w \in L_{2} \Longleftrightarrow \int_{0}^{a} w(x) d x_{2}=0$. So, $\left(d / d x_{1}\right) \int_{0}^{a} w(x) d x_{2}=0 \Longrightarrow \int_{0}^{a} w_{x_{1}}(x) d x_{2}=0 \Longrightarrow w_{x_{1}} \epsilon$ $L_{2}$. Hence $\int_{S} v_{x_{1}}\left(x_{1}\right) \overline{w_{x_{1}}(x)} d x=0$.

Now for all $u \in W_{2}^{1}(S), u=v+w, v \in \mathscr{H}_{1}, w \in \mathscr{H}_{2}$ one has

$$
\begin{aligned}
& \int_{S}|\nabla u(x)|^{2} d x \\
& =a \int_{\mathbb{R}}\left|v^{\prime}\left(x_{1}\right)\right|^{2} d x_{1}+\int_{S}|\nabla w(x)|^{2} d x \\
& \quad+\underbrace{\int_{S} \nabla v\left(x_{1}\right) \cdot \overline{\nabla w(x)} d x+\int_{S} \nabla w(x) \cdot \overline{\nabla v\left(x_{1}\right)} d x}_{=0} \\
& =a \int_{\mathbb{R}}\left|v^{\prime}\left(x_{1}\right)\right|^{2} d x_{1}+\int_{S}|\nabla w(x)|^{2} d x
\end{aligned}
$$

and

$$
\begin{aligned}
\int_{S} V(x)|u(x)|^{2} d x= & \int_{S} V(x)\left|v\left(x_{1}\right)\right|^{2} d x \\
& +\int_{S} V(x)|w(x)|^{2} d x \\
& +\int_{S} 2 V(x) \operatorname{Re}(v \cdot w) d x \\
\leq & 2 \int_{\mathbb{R}} \widetilde{V}\left(x_{1}\right)\left|v\left(x_{1}\right)\right|^{2} d x_{1} \\
& +2 \int_{S} V(x)|w(x)|^{2} d x,
\end{aligned}
$$

where

$$
\widetilde{V}\left(x_{1}\right)=\frac{1}{a} \int_{0}^{a} V(x) d x_{2} .
$$

So

$$
\begin{aligned}
& \int_{S}|\nabla u(x)|^{2} d x-\int_{S} V(x)|u(x)|^{2} d x \\
& \geq \int_{\mathbb{R}}\left|v^{\prime}\left(x_{1}\right)\right|^{2} d x_{1}-2 \int_{\mathbb{R}} \widetilde{V}\left(x_{1}\right)\left|v\left(x_{1}\right)\right|^{2} d x_{1} \\
& \quad+\int_{S}|\nabla w(x)|^{2} d x-2 \int_{S} V(x)|w(x)|^{2} d x .
\end{aligned}
$$


Hence

$$
N_{-}\left(q_{V, S}\right) \leq N_{-}\left(q_{1,2 \widetilde{V}, \mathbb{R}}\right)+N_{-}\left(q_{2,2 V, S}\right),
$$

where $q_{1,2 \widetilde{V}, \mathbb{R}}$ and $q_{2,2 V, S}$ denote the restrictions of the form $q_{2 V, S}$ to the spaces $\mathscr{H}_{1}$ and $\mathscr{H}_{2}$, respectively.

Let

$$
\begin{aligned}
& \mathscr{G}_{n}:=\int_{I_{n}}\left|x_{1}\right| \widetilde{V}\left(x_{1}\right) d x_{1}, \quad n \neq 0, \\
& \mathscr{G}_{0}:=\int_{I_{0}} \widetilde{V}\left(x_{1}\right) d x_{1} .
\end{aligned}
$$

Then similarly to the estimate before (39) in [6] one has

$$
N_{-}\left(q_{1,2 \widetilde{V}, \mathbb{R}}\right) \leq 1+7.61 \sum_{\left\{n \in \mathbb{Z}, \mathscr{G}_{n}>0.046\right\}} \sqrt{\mathscr{G}_{n}} .
$$

In terms of the original potential $V$

$$
\begin{aligned}
\mathscr{G}_{n} & =\int_{I_{n}}\left|x_{1}\right| \widetilde{V}\left(x_{1}\right) d x_{1} \\
& =\int_{I_{n}}\left|x_{1}\right|\left(\int_{0}^{a} V(x) d x_{2}\right) d x_{1} \\
& =\int_{I_{n} \times(0, a)}\left|x_{1}\right| V(x) d x
\end{aligned}
$$

and

$$
\begin{aligned}
\mathscr{G}_{0} & =\int_{I_{0}} \widetilde{V}\left(x_{1}\right) d x_{1}=\int_{I_{0}}\left(\int_{0}^{a} V(x) d x_{2}\right) d x_{1} \\
& =\int_{I_{0} \times(0, a)} V(x) d x .
\end{aligned}
$$

It now remains to find an estimate for $N_{-}\left(q_{2,2 V, S}\right)$ in (34).

Let $S_{n}:=J_{n} \times I, n \in \mathbb{Z}$, where $J_{n}$ and $I$ are as defined in (21). Then the variational principle (see, e.g., [11, Ch.6, $\$ 2.1$, Theorem 4]) implies

$$
N_{-}\left(q_{2,2 V, S}\right) \leq \sum_{n \in \mathbb{Z}} N_{-}\left(q_{2,2 V, S_{n}}\right),
$$

where

$$
\begin{aligned}
& q_{2,2 V, S_{n}}[w]:=\int_{S_{n}}|\nabla w(x)|^{2} d x \\
& \quad-2 \int_{S_{n}} V(x)|w(x)|^{2} d x, \\
& \operatorname{dom}\left(q_{2,2 V, S_{n}}\right)=\left\{w \in W_{2}^{1}\left(S_{n}\right)\right. \\
& \left.\cap L^{2}\left(S_{n}, V(x) d x\right): \int_{S_{n}} w(x) d x=0\right\} .
\end{aligned}
$$

Lemma 4 (cf. [6, Lemma 7.8]). There exists $C_{1}>0$ such that

$$
N_{-}\left(q_{2,2 V, S_{n}}\right) \leq C_{1}\|V\|_{L_{1}\left(J_{n}, L_{\mathscr{B}}(I)\right)}, \quad \forall V \geq 0
$$

(see (13)).
Proof. Let $I=\mathbb{~}$, the unit interval. Then it follows from [6, Lemma 7.7] that there is a constant $d_{1}>0$ such that

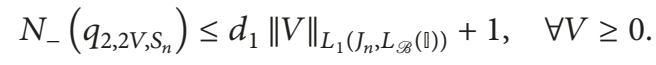

Similarly to (62) in [6] and using the Poincaré inequality (see, e.g., $[12,1.1 .11])$, there is a constant $d_{2}>0$ such that

$$
\begin{aligned}
& 2 \int_{S_{n}} V(x)|w(x)|^{2} d x \\
& \quad \leq d_{2}\|V\|_{L_{1}\left(J_{n}, L_{\mathscr{B}}(\mathbb{D})\right)} \int_{S_{n}}|\nabla w(x)|^{2} d x
\end{aligned}
$$

for all $w \in W_{2}^{1}\left(S_{n}\right) \cap L^{2}\left(S_{n}, V(x) d x\right)$ such that $\int_{S_{n}} w(x) d x=0$.

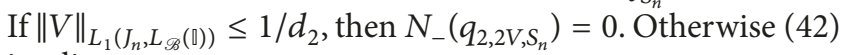
implies

$$
N_{-}\left(q_{2,2 V, S_{n}}\right) \leq C_{1}\|V\|_{L_{1}\left(J_{n}, L_{\mathscr{B}}(\mathbb{D})\right)}, \quad \forall V \geq 0,
$$

where $C_{1}:=d_{1}+d_{2}$. Hence (41) follows by the scaling $x_{2} \longrightarrow$ $a x_{2}$.

\section{Proof of Theorem 3}

Proof. If $\mathscr{D}_{n}<1 / C_{1}$, then $N_{-}\left(q_{2,2 V, S_{n}}\right)=0$ and one can drop this term from the sum (39). Hence, for any $c<1 / C_{1}$, (39) and Lemma 4 imply that

$$
N_{-}\left(q_{2,2 V, S}\right) \leq C_{1} \sum_{\left\{n \in \mathbb{Z}, \mathscr{D}_{n}>c\right\}} \mathscr{D}_{n} \quad \forall V \geq 0 .
$$

This together with (34) and (36) imply (22).

One can easily show that (22) is an improvement of the estimates obtained by A. Grigor'yan and N. Nadirashvili [3, Theorem 7.9] with a different $c$ and that (22) is strictly sharper. Indeed, let $B_{n}:=\|V\|_{\mathscr{B}, S_{n}}$. Then it follows from the embedding $L^{p}\left(S_{n}\right) \hookrightarrow L_{\mathscr{B}}\left(S_{n}\right)$ that there is a constant $C(p), p>1$ such that

$$
\begin{aligned}
B_{n} & =\|V\|_{\mathscr{B}, S_{n}} \leq C(p)\left(\int_{S_{n}} V(x)^{p} d x\right)^{1 / p} \\
& =C(p) b_{n}(V)
\end{aligned}
$$

where $b_{n}(V):=\left(\int_{S_{n}} V(x)^{p} d x\right)^{1 / p}$ (see [6, Remark 6.3]). Now it follows from the known theory of embeddings of mixednorm Orlicz spaces (see, e.g., $[13,14])$ that

$$
\mathscr{D}_{n} \leq C(p) b_{n}(V) \text {. }
$$

Hence

$$
N_{-}\left(q_{2,2 V, S_{n}}\right) \leq C_{2} b_{n}(V), \quad \forall V \geq 0,
$$

where $C_{2}:=C_{1} C(p)$. The scaling $V \longmapsto t V, t>0$, allows one to extend the above inequality to an arbitrary $V \geq 0$. Thus, for any $c<1 / C_{2}$, (22) implies [3, Theorem 7.9].

Next we will discuss different forms of (22). 
Remark 5. Note that

$$
\sum_{\left\{n \in \mathbb{Z}, \mathscr{G}_{n}>c\right\}} \sqrt{\mathscr{G}_{n}} \leq \frac{2}{\sqrt{c}}\left\|\left(\mathscr{G}_{n}\right)_{n \in \mathbb{Z}}\right\|_{1, w}
$$

(see (49) in [6]). Estimate (22) implies the following estimate:

$$
\begin{array}{r}
N_{-}\left(q_{V, S}\right) \leq 1+C_{3}\left(\left\|\left(\mathscr{G}_{n}\right)_{n \in \mathbb{Z}}\right\|_{1, w}+\|V\|_{L_{1}\left(\mathbb{R}, L_{\mathscr{B}}(I)\right)}\right) \\
\forall V \geq 0 .
\end{array}
$$

This follows from (49) and

$$
\begin{aligned}
\sum_{\left\{n \in \mathbb{Z}, \mathscr{D}_{n}>c\right\}} \mathscr{D}_{n} & \leq \sum_{n \in \mathbb{Z}} \mathscr{D}_{n}=\int_{\mathbb{R}}\left\|V\left(x_{1}, .\right)\right\|_{\mathscr{B}, I} d x_{1} \\
& =\|V\|_{L_{1}\left(\mathbb{R}, L_{\mathscr{B}}(I)\right)} .
\end{aligned}
$$

Equation (50) in turn implies the following:

$$
\begin{aligned}
& N_{-}\left(q_{V, S}\right) \leq 1+C_{4}\left(\left\|\left(\mathscr{G}_{n}\right)_{n \in \mathbb{Z}}\right\|_{1, w}\right. \\
& \left.+\int_{\mathbb{R}}\left(\int_{I}|V(x)|^{p} d x_{2}\right)^{1 / p} d x_{1}\right), \quad \forall V \geq 0,
\end{aligned}
$$

which is equivalent to

$$
\begin{aligned}
& N_{-}\left(q_{V, S}\right) \leq 1+C_{5}\left(\left\|\left(\mathscr{G}_{n}\right)_{n \in \mathbb{Z}}\right\|_{1, w}\right. \\
& \left.\quad+\int_{\mathbb{R}}\left(\int_{I}\left|V_{*}(x)\right|^{p} d x_{2}\right)^{1 / p} d x_{1}\right), \quad \forall V \geq 0,
\end{aligned}
$$

where $V_{*}(x)=V(x)-\widetilde{V}\left(x_{1}\right), \widetilde{V}\left(x_{1}\right)=(1 / a) \int_{0}^{a} V(x) d x_{2}$. Indeed,

$$
\begin{aligned}
& \mid \int_{\mathbb{R}}\left(\int_{I}|V(x)|^{p} d x_{2}\right)^{1 / p} d x_{1} \\
& \quad-\int_{\mathbb{R}}\left(\int_{I}\left|V_{*}(x)\right|^{p} d x_{2}\right)^{1 / p} d x_{1} \mid \\
& \quad \leq \int_{\mathbb{R}}\left(\int_{I}\left|\widetilde{V}\left(x_{1}\right)\right|^{p} d x_{2}\right)^{1 / p} d x_{1} \\
& \quad=a^{1 / p} \int_{\mathbb{R}} \widetilde{V}\left(x_{1}\right) d x_{1} \\
& \quad=a^{1 / p} \sum_{n \in \mathbb{Z}} \int_{I_{n} \times(0, a)} V(x) d x \leq a^{1 / p} \sum_{n \in \mathbb{Z}} 2^{-|n|+1} \mathscr{G}_{n} \\
& \quad \leq \text { const } \sup _{n \in \mathbb{Z}} \mathscr{G}_{n} \leq \text { const } \|\left(\mathscr{G}_{n}\right)_{n \in \mathbb{Z} \|_{1, w}} .
\end{aligned}
$$

Similarly,

$$
\begin{aligned}
& \left|\|V\|_{L_{1}\left(\mathbb{R}, L_{\mathscr{B}}(I)\right)}-\left\|V_{*}\right\|_{L_{1}\left(\mathbb{R}, L_{\mathscr{B}}(I)\right)}\right| \\
& \quad=\left|\int_{\mathbb{R}}\left\|V\left(x_{1}, \cdot\right)\right\|_{\mathscr{B}, I} d x_{1}-\int_{\mathbb{R}}\left\|V_{*}\left(x_{1}, \cdot\right)\right\|_{\mathscr{B}, I} d x_{1}\right| \\
& \quad \leq \int_{\mathbb{R}}\left\|\widetilde{V}\left(x_{1}\right)\right\|_{\mathscr{B}, I} d x_{1}=\mathrm{const} \int_{\mathbb{R}}\left|\widetilde{V}\left(x_{1}\right)\right| d x_{1} \\
& \quad \leq \mathrm{const} \sup _{n \in \mathbb{Z}} \mathscr{G}_{n} \leq \mathrm{const}\left\|\left(\mathscr{G}_{n}\right)_{n \in \mathbb{Z}}\right\|_{1, w} .
\end{aligned}
$$

Hence (50) is equivalent to the following estimate:

$$
\begin{array}{r}
N_{-}\left(q_{V, S}\right) \leq 1+C_{6}\left(\left\|\left(\mathscr{G}_{n}\right)_{n \in \mathbb{Z}}\right\|_{1, w}+\left\|V_{*}\right\|_{L_{1}\left(\mathbb{R}, L_{\mathscr{B}}(I)\right)}\right), \\
\forall V \geq 0 .
\end{array}
$$

Note the last term on the right-hand side of (56) (and (53)) drops out if $V$ does not depend on $x_{2}$.

Let $\alpha>0$ be given. It is well known that the lowest possible (semiclassical) rate of growth of $N_{-}\left(q_{\alpha V, S}\right)$ is

$$
N_{-}\left(q_{\alpha V, S}\right)=O(\alpha) \quad \text { as } \alpha \longrightarrow+\infty
$$

(see, e.g., $[2,4])$. It turns out that the finiteness of the first term in (22) is necessary for $N_{-}\left(q_{\alpha V, S}\right)=O(\alpha)$ as $\alpha \longrightarrow+\infty$ to hold (see the next theorem).

Theorem 6. Let $V \geq 0$. If $N_{-}\left(q_{\alpha V, S}\right)=O(\alpha)$ as $\alpha \longrightarrow+\infty$, then $\left\|\mathscr{G}_{n}\right\|_{1, w}<\infty$.

Proof. Consider the function

$$
\begin{aligned}
& w_{n}\left(x_{1}\right) \\
& := \begin{cases}0, & x_{1} \leq 2^{n-1} \text { or } x_{1} \geq 2^{n+2}, \\
4\left(x_{1}-2^{n-1}\right), & 2^{n-1}<x_{1}<2^{n}, \\
2^{n+1}, & 2^{n} \leq x_{1} \leq 2^{n+1}, \\
2^{n+2}-x_{1}, & 2^{n+1}<x_{1}<2^{n+2},\end{cases}
\end{aligned}
$$

for $n>0$. Let $u_{n}(x)=w_{n}\left(x_{1}\right) u_{1}\left(x_{2}\right)$, where $u_{1}$ is the first eigenfunction of the one-dimensional second-order differential operator on $L^{2}((0, a))$ which is identically equal to 1 . Then we have

$$
\begin{aligned}
& \int_{S}\left|\nabla u_{n}(x)\right|^{2} d x=a \int_{\mathbb{R}}\left|w_{n}^{\prime}\left(x_{1}\right)\right|^{2} d x_{1} \\
& =a\left(\int_{2^{n-1}}^{2^{n}}\left|w_{n}^{\prime}\left(x_{1}\right)\right|^{2} d x_{1}+\int_{2^{n}}^{2^{n+1}}\left|w_{n}^{\prime}\left(x_{1}\right)\right|^{2} d x_{1}\right. \\
& \left.+\int_{2^{n+1}}^{2^{n+2}}\left|w_{n}^{\prime}\left(x_{1}\right)\right|^{2} d x_{1}\right)=C_{7} 2^{n+1}
\end{aligned}
$$

where

$$
C_{7}:=5 a
$$


Now

$$
\begin{aligned}
& \int_{S} V(x)\left|u_{n}(x)\right|^{2} d x \geq \int_{2^{n}}^{2^{n+1}} 2^{2(n+1)} d x_{1} \int_{0}^{a} V(x) d x_{2} \\
& \geq \int_{2^{n}}^{2^{n+1}} 2^{n+1} d x_{1} \int_{0}^{a}\left|x_{1}\right| V(x) d x_{2} \\
& \quad=\frac{1}{C_{7}}\left(\int_{S}\left|\nabla u_{n}(x)\right|^{2} d x\right) \mathscr{G}_{n} .
\end{aligned}
$$

If $\mathscr{G}_{n}>C_{7}$, then $q_{V, S}\left[u_{n}\right]<0$. The auxiliary functions $w_{n}$ can be defined similarly for $n \leq 0$. Since $u_{n}$ and $u_{k}$ have disjoint supports for $|n-k| \geq 3$, then

$$
N_{-}\left(q_{V, S}\right) \geq \frac{1}{3} \operatorname{card}\left\{n \in \mathbb{Z}: \mathscr{G}_{n}>C_{7}\right\}
$$

(see [6, Theorem 9.1]). If, for some constant $C_{8}>0$, $N_{-}\left(q_{\alpha V, S}\right) \leq C_{8} \alpha$, then

$$
\frac{1}{3} \operatorname{card}\left\{n \in \mathbb{Z}: \alpha \mathscr{G}_{n}>C_{7}\right\} \leq C_{8} \alpha
$$

and so

$$
\operatorname{card}\left\{n \in \mathbb{Z}: \mathscr{G}_{n}>\frac{C_{7}}{\alpha}\right\} \leq 3 C_{8} \alpha .
$$

With $s=C_{7} / \alpha$ we have

$$
\operatorname{card}\left\{n \in \mathbb{Z}: \mathscr{G}_{n}>s\right\} \leq C_{9} s^{-1}, \quad s>0,
$$

where $C_{9}:=3 C_{7} C_{8}$.

\section{Estimates Involving Norms of the Potential Supported by a Lipschitz Curve inside a Strip}

In this section we obtain estimates analogous to those in the previous section when the potential $V$ is strongly singular, i.e., when $V$ is supported by a Lipschitz curve $\ell$ embedded in $S$. When dealing with function spaces on $\ell$, we will always assume that $\ell$ is equipped with the arc length measure. Before we introduce the estimates, let us first look at the following operator that we shall need in the sequel.

Consider a one-dimensional Schrödinger operator $H_{X, \alpha}$, with point $\delta$-interactions on a countable set $X=\left\{x_{k}\right\}_{k=1}^{\infty}$ of points, called points of interaction, and intensities $\alpha=$ $\left\{\alpha_{k}\right\}_{k=1}^{\infty}$, defined by the differential expression $-d^{2} / d x^{2}$ on functions $w(x)$ that belong to the Sobolev space $W_{2}^{2}(\mathbb{R} \backslash X)$ satisfying, in the points of the set $X$, the following conjugation conditions:

$$
\begin{array}{r}
w\left(x_{k}+0\right)=w\left(x_{k}-0\right), \\
w^{\prime}\left(x_{k}+0\right)-w^{\prime}\left(x_{k}-0\right)=\alpha_{k} w\left(x_{k}\right) .
\end{array}
$$

Since, for each $k,\left(x_{k}, x_{k+1}\right)$ is an open interval, then any function in $W_{2}^{2}\left(\left(x_{k}, x_{k+1}\right)\right)$ and its derivative have well defined (one-sided) values at the end-points. The operator $H_{X, \alpha}$ has the following representation:

$$
H_{X, \alpha}:=-\frac{d^{2}}{d x^{2}}+\sum_{k=1}^{\infty} \alpha_{k} \delta\left(x-x_{k}\right),
$$

where $\delta$ is Dirac's delta distribution. We shall assume that $H_{X, \alpha}$ is self-adjoint (see, e.g., [15] in case the set $X$ is finite). One can also define operator (67) via its quadratic form as follows:

$$
q[w]:=\int_{\mathbb{R}}\left|w^{\prime}(x)\right|^{2} d x-\sum_{k=1}^{\infty} \alpha_{k}\left|w\left(x_{k}\right)\right|^{2},
$$

$$
\forall w \in W_{2}^{1}(\mathbb{R}) .
$$

Lemma 7. Given an infinite sequence of positive numbers $\left(\alpha_{k}\right)$, there is a sequence of points $\left(x_{k}\right)$ in $X$ such that (67) has infinitely many negative eigenvalues.

Proof. Let $\psi \in C_{0}^{\infty}(\mathbb{R})$ such that

$$
\psi(x)= \begin{cases}1 & \text { if }|x|<\frac{1}{2} \\ 0 & \text { if }|x| \geq 1\end{cases}
$$

Assume that $x_{k+1}-x_{k}>x_{k}-x_{k-1}$, for all $k=1,2, \ldots$ and let $\varphi_{k}(x):=\psi\left(\left(2 /\left(x_{k}-x_{k-1}\right)\right)\left(x-x_{k}\right)\right)$. Then

$$
\varphi_{k}(x)= \begin{cases}1 & \text { if }\left|x-x_{k}\right|<\frac{x_{k}-x_{k-1}}{4}, \\ 0 & \text { if }\left|x-x_{k}\right| \geq \frac{x_{k}-x_{k-1}}{2} .\end{cases}
$$

Let $\mathscr{L}$ be a linear subspace of $W_{2}^{1}(\mathbb{R})$ defined by

$$
\mathscr{L}:=\left\{w \in W_{2}^{1}(\mathbb{R}): w=\sum_{k=1}^{\infty} a_{k} \varphi_{k}, a_{k} \in \mathbb{C}\right\} .
$$

Since $\varphi_{k}$ and $\varphi_{j}$ for $k \neq j$ have disjoint supports, then $\operatorname{dim} \mathscr{L}=\infty$. Thus, for all $w \in \mathscr{L} \backslash\{0\}$, it follows from (68) that

$$
\begin{aligned}
& q[w]=\sum_{k=1}^{\infty}\left|a_{k}\right|^{2}\left(\int_{\mathbb{R}}\left|\varphi_{k}^{\prime}(x)\right|^{2} d x-\alpha_{k}\right)=\sum_{k=1}^{\infty}\left|a_{k}\right|^{2} \\
& \cdot\left(\frac{4}{\left|x_{k}-x_{k-1}\right|^{2}} \int_{\mathbb{R}}\left|\psi^{\prime}\left(\frac{2}{x_{k}-x_{k-1}}\left(x-x_{k}\right)\right)\right|^{2} d x\right. \\
& \left.-\alpha_{k}\right)=\sum_{k=1}^{\infty}\left|a_{k}\right|^{2}\left(\frac{2}{\left|x_{k}-x_{k-1}\right|} \int_{\mathbb{R}}\left|\psi^{\prime}(t)\right|^{2} d t\right. \\
& \left.-\alpha_{k}\right),
\end{aligned}
$$

where $t=\left(2 /\left(x_{k}-x_{k-1}\right)\right)\left(x-x_{k}\right)$. Take $x_{k}$ such that

$$
x_{k}-x_{k-1}>\frac{2}{\alpha_{k}} \int_{\mathbb{R}}\left|\psi^{\prime}(t)\right|^{2} d t,
$$

then $q[w]<0$ and operator (67) has infinitely many negative eigenvalues. 
Let us now return to operator (1) with $V$ supported by and locally integrable on a Lipschitz curve $\ell$ embedded in $S$. Let

$$
\begin{aligned}
q_{V, \ell}[u]:= & \int_{S}|\nabla u(x)|^{2} d x \\
& -\int_{\ell} V(x)|u(x)|^{2} d s(x) \\
\operatorname{dom}\left(q_{V, \ell}\right)= & W_{2}^{1}(S) \cap L^{2}(\ell, V d s) .
\end{aligned}
$$

Let $\left\{x_{1}^{(k)}\right\}, k \in \mathbb{N}$ be a sequence of points on $\mathbb{R}$ satisfying conditions in (66). Define

$$
\gamma_{k}:=\ell \cap\left(\left\{x_{1}^{(k)}\right\} \times(0, a)\right), \quad x_{1} \in \mathbb{R}, k \in \mathbb{N}
$$

and

$$
\Sigma:=\left\{x_{1}^{(k)}:\left|\gamma_{k}\right|>0\right\}
$$

Then the set $\Sigma$ is at most countable. Let $I$ be an arbitrary interval in $\mathbb{R}$ and let

$$
v(I):=\int_{\ell \cap(I \times(0, a))} V(x) d s(x) .
$$

Furthermore, let

$$
\begin{aligned}
\mathscr{F}_{n} & :=\int_{I_{n}}\left|x_{1}\right| d v\left(x_{1}\right), \quad n \neq 0, \\
\mathscr{F}_{0} & :=\int_{I_{0}} d v\left(x_{1}\right) \quad(c f .(35)), \\
\ell_{n} & :=\ell \cap S_{n}, \quad S_{n}:=(n, n+1) \times(0, a), n \in \mathbb{N}, \\
\mathscr{C}_{n} & :=\|V\|_{\mathscr{B}, \ell_{n}}^{\text {(av) }} .
\end{aligned}
$$

Theorem 8. Suppose that $N$ is the cardinality of $\Sigma$. Then there exist constants $c_{1}, c_{2}, C_{10}, C_{11}>0$ such that

$$
\begin{aligned}
N_{-}\left(q_{V, \ell}\right) \leq 1 & +N+C_{9} \sum_{\left\{n \in \mathbb{Z}, \mathscr{F}_{n}>\mathcal{C}_{1}\right\}} \sqrt{\mathscr{F}_{n}} \\
& +C_{10} \sum_{\left\{n \in \mathbb{Z}, \mathscr{C}_{n}>\mathcal{C}_{2}\right\}} \mathscr{C}_{n}, \quad \forall V \geq 0 .
\end{aligned}
$$

If $\sum$ is infinite, then $N_{-}\left(q_{V, \ell}\right)=\infty$.

Proof. Let $q_{1,2 V, \ell}$ and $q_{2,2 V, \ell}$ be the restrictions of the form $q_{V, \ell}$ to the subspaces $\mathscr{H}_{1}$ and $\mathscr{H}_{2}$, respectively (see (27)), and then

$$
\left.N_{-}\left(q_{V, \ell}\right) \leq N_{-}\left(q_{1,2 V, \ell}\right)\right)+N_{-}\left(q_{2,2 V, \ell}\right)
$$

(cf. (34)). Let us start by estimating the first term on the righthand side of (80). On the complement of $\Sigma, v\left(\left\{x_{1}\right\}\right)=0$ for all $x_{1} \in \mathbb{R}$. This implies

$$
\begin{aligned}
& \int_{\ell} V(x)|u(x)|^{2} d s(x) \\
& =\int_{\mathbb{R}}\left|w\left(x_{1}\right)\right|^{2} d v\left(x_{1}\right) \\
& \quad+\left|w\left(x_{1}\right)\right|^{2} \sum_{k \in \mathbb{N}} \int_{\gamma_{k}} V\left(x_{2}\right) d x_{2} \\
& =\int_{\mathbb{R}}\left|w\left(x_{1}\right)\right|^{2} d v\left(x_{1}\right)+\sum_{k \in \mathbb{N}} c_{k}\left|w\left(x_{1}\right)\right|^{2},
\end{aligned}
$$

where $c_{k}:=\int_{\gamma_{k}} V\left(x_{2}\right) d x_{2}<\infty$. Hence

$$
\begin{aligned}
q_{1,2 V, \ell}[u]= & \int_{S}|\nabla u(x)|^{2} d x-2 \int_{\ell} V(x)|u(x)|^{2} d x \\
= & \int_{\mathbb{R}}\left|w^{\prime}\left(x_{1}\right)\right|^{2} d x_{1} \\
& -2 \int_{\mathbb{R}}\left|w\left(x_{1}\right)\right|^{2} d \nu\left(x_{1}\right) \\
& -\sum_{k \in \mathbb{N}} c_{k}^{\prime}\left|w\left(x_{1}\right)\right|^{2},
\end{aligned}
$$

where $c_{k}^{\prime}:=2 c_{k}$. Let

$$
\begin{aligned}
q_{1,2 v}[w]:= & \int_{\mathbb{R}}\left|w^{\prime}\left(x_{1}\right)\right|^{2} d x_{1} \\
& -2 \int_{\mathbb{R}}\left|w\left(x_{1}\right)\right|^{2} d v\left(x_{1}\right), \\
\operatorname{dom}\left(q_{1,2 v}\right)= & W_{2}^{1}(\mathbb{R}) \cap L^{2}(\mathbb{R}, d v), \\
q_{2, c_{k}^{\prime}}[w]:= & \int_{\mathbb{R}}\left|w^{\prime}\left(x_{1}\right)\right|^{2} d x_{1}-\sum_{k \in \mathbb{N}} c_{k}^{\prime}\left|w\left(x_{1}\right)\right|^{2}, \\
\operatorname{dom}\left(q_{2, c_{k}^{\prime}}\right)= & W_{2}^{1}(\mathbb{R}) .
\end{aligned}
$$

Then, it follows from [3, Lemma 3.6] that

$$
N_{-}\left(q_{1,2 V, \ell}\right) \leq N_{-}\left(q_{1,2 v}\right)+N_{-}\left(q_{2, c_{k}^{\prime}}\right) .
$$

Similarly to (36) one has

$$
N_{-}\left(q_{1,2 v}\right) \leq 1+7.16 \sum_{\left\{n \in \mathbb{Z}, \mathscr{F}_{n}>0.046\right\}} \sqrt{\mathscr{F}_{n}} .
$$

If $\Sigma$ is finite, then

$$
N_{-}\left(q_{2, c_{k}^{\prime}}\right) \leq N
$$

(see, e.g., [15]). Otherwise, Lemma 7 implies

$$
N_{-}\left(q_{2, c_{k}^{\prime}}\right)=\infty
$$


Now, it remains to estimate the second term on the right-hand side of (80). Let

$$
\begin{aligned}
& q_{2,2 V, \ell_{n}}[u] \\
& \quad:=\int_{S_{n}}|\nabla u(x)|^{2} d x-\int_{\ell_{n}} V(x)|u(x)|^{2} d s(x) \\
& \operatorname{dom}\left(q_{2,2 V, \ell_{n}}\right) \\
& \quad=\left\{u \in W_{2}^{1}\left(S_{n}\right) \cap L^{2}\left(\ell_{n}, V d s\right): \int_{S_{n}} u(x) d x=0\right\} .
\end{aligned}
$$

For any $V \in L_{\mathscr{B}}\left(\ell_{n}\right), V \geq 0$ and any $r \in \mathbb{N}$, following a similar argument in the proof of [6, Lemma 7.6], one can show that there exists a finite cover of $\ell_{n}$ by rectangles $S_{n_{k}}, k=1, \ldots, r_{0}$ such that $r_{0} \leq r$ and

$$
\begin{aligned}
& \int_{\ell_{n}} V(x)|u(x)|^{2} d s(x) \\
& \quad \leq C_{12} r^{-1}\|V\|_{\mathscr{B}, \ell_{n}}^{(\mathrm{av})} \int_{S_{n}}|\nabla u(x)|^{2} d x
\end{aligned}
$$

for all $u \in W_{2}^{1}\left(S_{n}\right) \cap L^{2}\left(\ell_{n}, V d s\right)$ with $\int_{S_{n_{k}}} u(x) d x=0$, where $C_{12}$ is a constant independent of $V$. Now, using (89) instead of [6, Lemma 7.6] in the proof of [6, Lemma 7.7], one can easily show similarly to [6, Lemma 7.8] that there is a constant $C_{11}>$ 0 such that

$$
N_{-}\left(q_{2,2 V, \ell_{n}}\right) \leq C_{11}\|V\|_{\mathscr{B}, \ell_{n}}^{(\text {av) }}, \quad \forall V \geq 0
$$

If $\|V\|_{\mathscr{B}, \ell_{n}}^{(\text {av }}<1 / C_{11}$, then $N_{-}\left(q_{2,2 V, \ell_{n}}\right)=0$. Thus, similarly to (39) one has for any $c_{2}<1 / C_{11}$ that

$$
N_{-}\left(q_{2,2 V, \ell}\right) \leq C_{11} \sum_{\left\{\mathscr{C}_{n}>c_{2}, n \in \mathbb{Z}\right\}} \mathscr{C}_{n}, \quad \forall V \geq 0
$$

Hence, the statement of the theorem follows from (80), (84), (85), (86), (87), and (91).

\section{Data Availability}

No data were used to support this study.

\section{Conflicts of Interest}

The author declares that he has no conflicts of interest.

\section{Acknowledgments}

This research was supported by the Commonwealth Scholarship Commission in the United Kingdom, grant UGCA-2013138.

\section{References}

[1] G. V. Rozenbljum, "Distribution of the discrete spectrum of singular differential operators," Doklady Akademii Nauk SSSR, vol. 202, pp. 1012-1015, 1972.
[2] M. S. Birman and A. Laptev, "The negative discrete spectrum of a two-dimensional Schrödinger operator," Communications on Pure and Applied Mathematics, vol. 49, no. 9, pp. 967-997, 1996.

[3] A. Grigor'yan and N. Nadirashvili, "Negative eigenvalues of two-dimensional Schrödinger operators," Archive for Rational Mechanics and Analysis, vol. 217, no. 3, pp. 975-1028, 2015.

[4] A. Laptev and M. Solomyak, "On spectral estimates for twodimensional Schrödinger operators," Journal of Spectral Theory, vol. 3, no. 4, pp. 505-515, 2013.

[5] R. A. Adams, Sobolev Spaces, Academic Press, New York, NY, USA, 1975.

[6] E. Shargorodsky, "On negative eigenvalues of two-dimensional Schrödinger operators," Proceedings of the London Mathematical Society. Third Series, vol. 108, no. 2, pp. 441-483, 2014.

[7] M. Solomyak, "Piecewise-polynomial approximation of functions from $H^{l}\left((0,1)^{d}\right), 2 \mathrm{l}=\mathrm{d}$, and applications to the spectral theory of the Schrödinger operators," Israel Journal of Mathematics, vol. 86, no. 1-3, pp. 253-275, 1994.

[8] R. L. Frank and A. Laptev, "Bound on the number of negative eigenvalues of two-dimensional Schrödinger operators on domains," https://arxiv.org/abs/1712.03167.

[9] M. Karuhanga, "On estimates for the number of negative eigenvalues of two-dimensional Schrödinger operators with potentials supported by Lipschitz curves," Journal of Mathematical Analysis and Applications, vol. 456, no. 2, pp. 1365-1379, 2017.

[10] M. S. Birman and M. Z. Solomjak, Spectral Theory of Self-Adjoint Operators in Hilbert Space, Dordrecht etc., Kluwer, 1987.

[11] R. Courant and D. Hilbert, Methods in Mathematical Physics, vol. 1, Interscience Publishers. Inc, New York, 1966.

[12] V. G. Mazya, Sobolev Spaces. With Applications to Elliptic Partial Differential Equations, Springer, Berlin-Heildelberg, 2011.

[13] A. Boccuto, A. V. Bukhvalov, and A. R. Sambucini, "Some inequalities in classical spaces with mixed norms," Positivity. An International Journal devoted to the Theory and Applications of Positivity in Analysis, vol. 6, no. 4, pp. 393-411, 2002.

[14] B. Firlej and W. Matuszewska, "Some remarks on spaces provided with mixed norm," Comment.Math.Prace Mat, vol. 17, pp. 347-357, 1974.

[15] S. Albeverio, F. Gesztesy, R. Høegh-Krohn, and H. Holden, Solvable Models in Quantum Mechanics, Springer, 1988. 


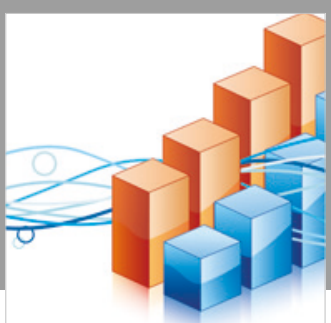

Advances in

Operations Research

\section{-n-m}
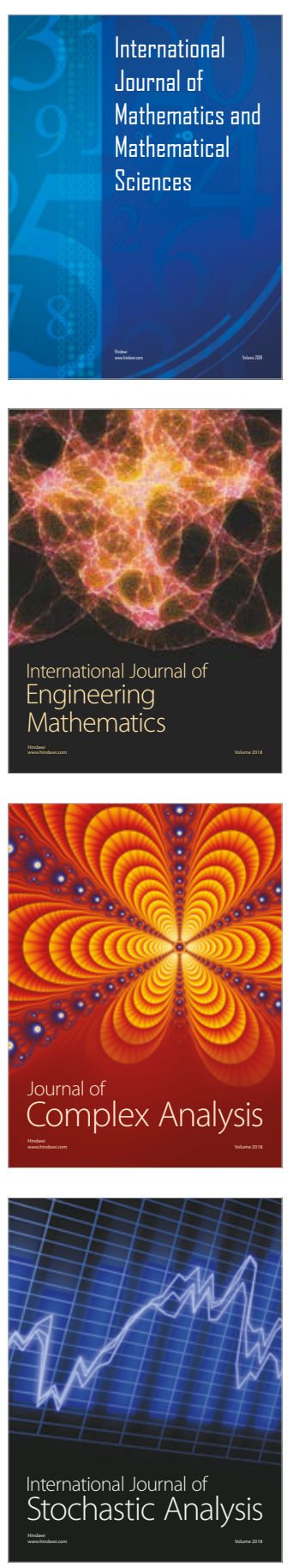
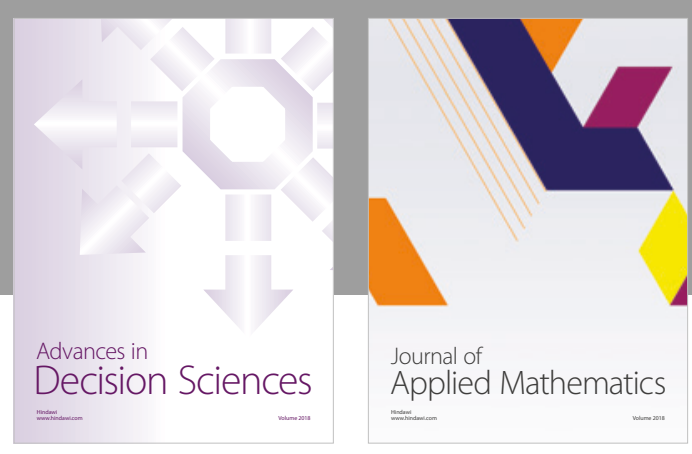

Journal of

Applied Mathematics
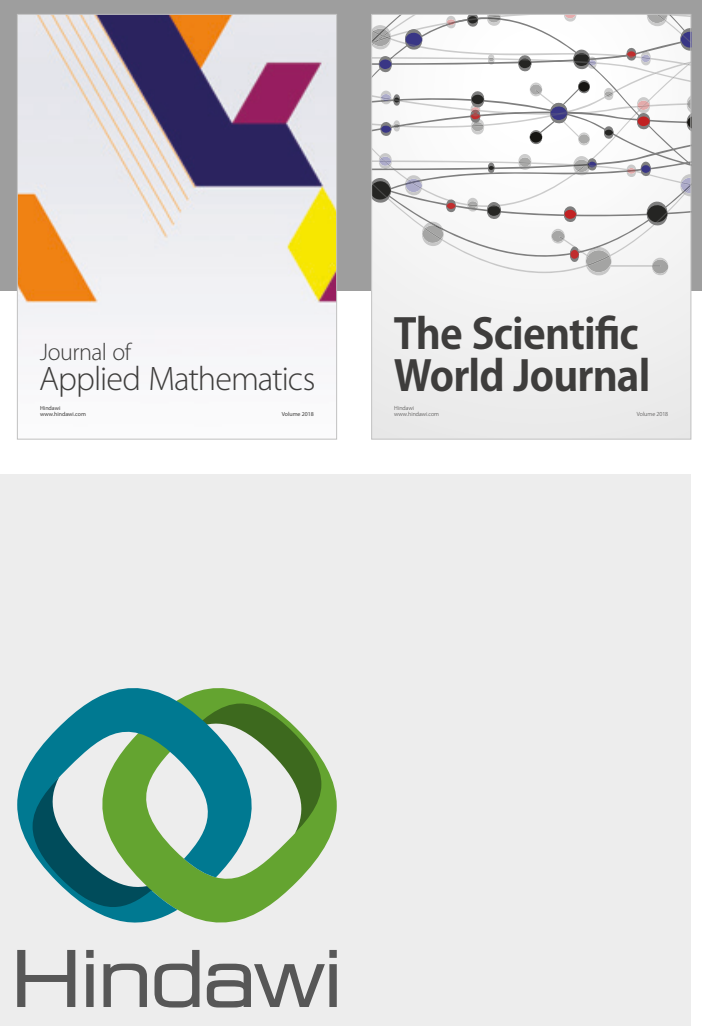

Submit your manuscripts at

www.hindawi.com

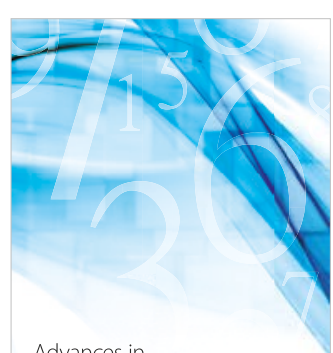

Advances in
Numerical Analysis
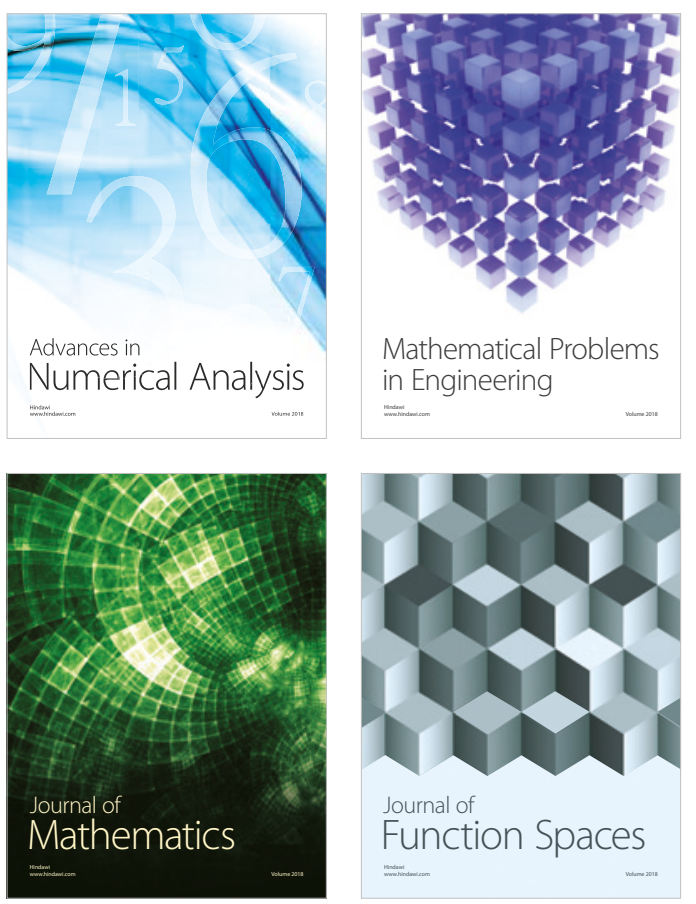

Mathematical Problems in Engineering

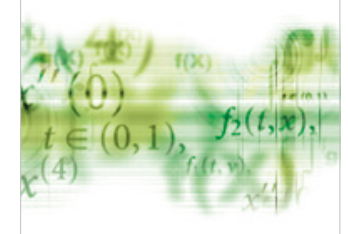

International Journal of

Differential Equations

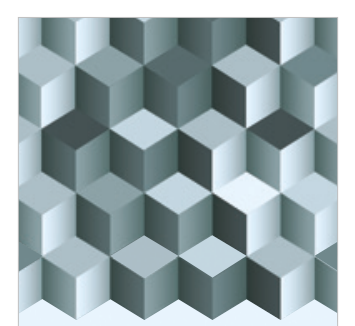

Journal of

Function Spaces

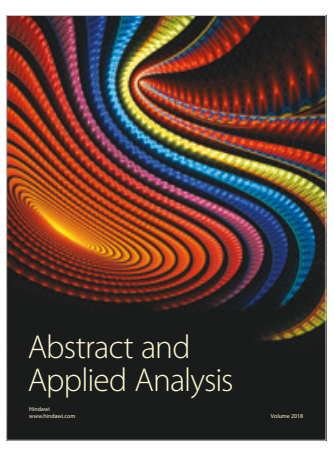

The Scientific

World Journal

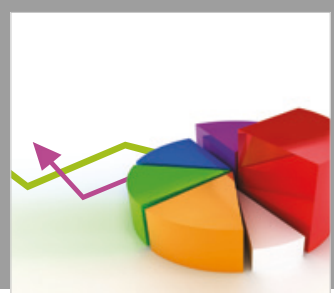

Journal of

Probability and Statistics
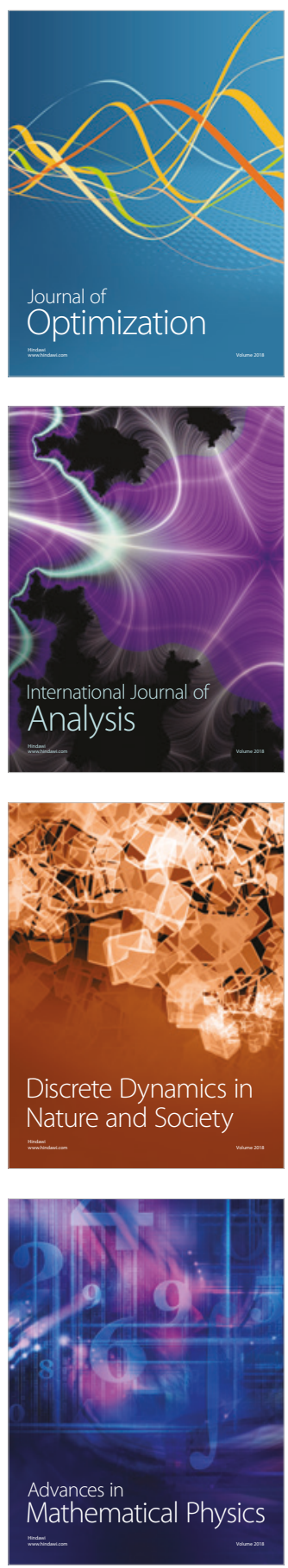